\title{
Educação continuada: experiência na rede SUS da região central de São Paulo
}

\section{Continuing education: experience in the SUS network in the central region of São Paulo}

\section{Maria Luiza Ferreira De Barba' (D) Gabriela Furst Vaccarezza² (D) Carolina Felipe Soares Brandão ${ }^{3}$ (D) Nivaldo Carneiro Junior ${ }^{4}$ (D)}

${ }^{1}$ Instituto de Atenção Básica e Avançada à Saúde (São Paulo). Universidade Estácio de Sá (Rio de Janeiro). São Paulo/Rio de Janeiro, Brasil. marialuizadebarba@hotmail.com ${ }^{2}$ Instituto de Atenção Básica e Avançada à Saúde (São Paulo), Universidade Municipal de São Caetano do Sul (São Caetano do Sul). São Paulo, Brasil. gavaccarezza@gmail.com ${ }^{3}$ Autora para correspondência. Universidade Municipal de São Caetano do Sul (São Caeatano do Sul), Universidade Cidade de São Paulo (São Paulo). São Paulo, Brasil. carolinafs11@gmail.com ${ }^{4}$ Faculdade de Ciências Médicas da Santa Casa de São Paulo (São Paulo). São Paulo, Brasil. nicarneirojr@uol.com.br

RESUMO | INTRODUÇÃO: A efetiva implantação do cuidado integral à população dos serviços de saúde faz-se necessário pensar na qualificação dos profissionais e processos de trabalho por meio de atividades dialógicas com base na troca de conhecimentos e práticas de forma engajada e no reconhecimento das singularidades vividas no cotidiano do serviço, possibilitando, assim, aquisição de novas habilidades, processos que desafiam a consolidação do SUS. OBJETIVO: Descrever o processo de formação desenvolvido por meio da Educação Continuada em atendimentos de urgências e emergências, na qualificação dos profissionais médicos e enfermeiros na Coordenadoria Regional de Saúde Centro do Município de São Paulo. METOdologiA: Estudo descritivo do tipo relato de experiência a partir das condutas de capacitações em saúde conduzidas através da educação continuada baseadas em metodologias ativas de aprendizagem. RESULTADOS: A partir do diagnóstico situacional foi identificado a necessidade de realizar atividades voltadas ao atendimento de situações mais focadas em urgência e emergências clínicas. Dessa forma, foram propostos treinamentos para os médicos e enfermeiros destes serviços conforme temáticas discutidas com a equipe e coordenação das unidades. CONCLUSÃo: A formulação e utilização dos mecanismos de gestão e avaliação baseados no planejamento estratégico e análise de indicadores de monitoramento e desempenho, propiciaram a criação de treinamentos baseados em metodologias ativas de aprendizagem com temáticas específicas direcionadas a estes grupos de profissionais.

PALAVRAS-CHAVE: Treinamento por simulação. Educação em saúde. Saúde pública. Aprendizagem ativa.
ABSTRACT | INTRODUCTION: The effective implementation of comprehensive care to the population of health services makes it necessary to think about the qualification of professionals and work processes through dialogical activities based on the exchange of knowledge and practices in an engaged way and the recognition of the singularities experienced in the daily life of the patient. thus enabling the acquisition of new skills, processes that challenge the consolidation of SUS. OBJECTIVE: Describing the training process developed through Continuing Education in urgent and emergency care in the qualification of medical professionals and nurses in the Regional Health Coordination Center of the Municipality of São Paulo. METHODOLOGY: Descriptive study of the type experience report based on the conduct of health training conducted through continuing education based on active learning methodologies. RESULTS: Based on the situational diagnosis, the need to carry out activities aimed at meeting situations more focused on urgency and clinical emergencies was identified. Thus, training was proposed for doctors and nurses in these services, according to topics discussed with the team and coordination of the units. CONCLUSION: The formulation and use of management and evaluation mechanisms based on strategic planning and analysis of monitoring and performance indicators, led to the creation of training based on active learning methodologies with specific themes aimed at these groups of professionals.

KEYWORDS: Simulation training. Health education. Public health. Active learning. 


\section{Introdução}

Com os desafios impostos para efetiva implantação do cuidado integral em saúde, é necessário pensar na qualificação dos profissionais e dos processos de trabalho por meio de atividades dialógicas, com base na troca de conhecimentos e práticas, propiciando o engajamento dos mesmos e a aplicabilidade prática dos assuntos abordados. É fundamental que o participante consiga identificar na estratégia de ensino-aprendizagem empregada o reconhecimento das singularidades vividas no seu cotidiano de trabalho, como também situações adversas que o desafiem a adquirir novas habilidades. Ademais, é relevante no processo de trabalho das equipes de saúde, que o profissional tenha consciência de seu comprometimento pessoal, profissional e social com o meio ao qual está inserido e busque melhorias em sua formação; de forma que esteja em contínua construção, constituindo um processo e uma ferramenta para complementar a formação do sujeito como um todo¹. Desse modo, educação continuada é uma estratégia potente, pois vai além da capacitação profissional, isto é, insere-se na realidade do trabalho a partir de uma contextualização do equipamento de saúde ${ }^{2}$.

Nessa perspectiva, o modelo teórico-metodológico do que se denomina de construtivismo exerce importante contribuição para a formação/qualificação profissional, pois fundamenta-se em estratégias problematizadoras de ensino-aprendizagem. A tendência atual nos currículos em saúde e departamentos de educação continuada são modelos híbridos, ou seja, diversas estratégias complementares que objetivam atender a andragogia, ou seja, potencializar a aprendizagem através do reconhecimento pelo participante do motivo pelo qual ele deve aprender determinado assunto ${ }^{3}$. Dentro das várias vertentes atuais e que cresce a cada momento, tem-se a Aprendizagem Baseada em Equipes (ABE) conhecida como TBL (Team Based Learning), que pode ser definida como uma estratégia de aprendizagem por metodologias ativas, importante ferramenta para o processo de trabalho em equipe ${ }^{4}$.
$\mathrm{Na} A B E$, o responsável pela atividade seleciona previamente o material disparador da temática que será objeto da sessão e distribui pelos grupos. Após uma breve exposição para a significação da temática que será discutida, é aplicado um teste a ser resolvido inicialmente de forma individual e a seguir coletivamente, também conhecidos, respectivamente, como Teste de Garantia de Prontidão e Votação Coletiva4. As respostas são computadas individual e coletivamente. Esta etapa tem por objetivo avaliar se o material distribuído previamente foi objeto de reflexão, bem como, na fase de discussão em grupos, a capacidade dos estudantes gerarem consenso, por leituras complementares realizadas, por conhecimento prévio ou outra forma de aprendizagem.

Em sequência, frente aos resultados alcançados e a declaração das hipóteses consideradas corretas pelo facilitador, ocorre a discussão dos tópicos e das respostas obtidas, também denominada de Fase de Argumentação, sempre voltadas a uma realidade perceptível pelos estudantes como significativa, frente à sua formação profissional ${ }^{5-7}$. Neste momento é possível que os participantes defendam divergências das respostas consideradas corretas. Por último, o facilitador apresenta uma situação da prática profissional na mesma temática das discussões, onde os participantes deverão fornecer subsídios para a formulação de hipóteses diagnósticas, utilizando-se do raciocínio clínico, bem como condutas aplicáveis à investigação clínica e complementar e ao plano terapêutico adequado 5 .

Outra metodologia ativa de aprendizagem utilizada é a simulação clínica. A simulação pode ser definida como uma estratégia educacional que replica experiências da vida real através de cenários clínicos que favoreçam um ambiente seguro, participativo e de interatividade, onde pode ser utilizados desde manequins para treino de habilidades procedimentais, atores como pacientes simulados para capacitações procedimentais, mas também comportamentais, e robôs de alta fidelidade que possibilitam diversas manipulações com respostas imediatas as intervenções e que podem associar todos os níveis de desempenhos esperados dos profissionais ${ }^{6}$. 
No ensino e capacitação do atendimento as emergências clínicas em geral, a relação entre a possibilidade de aprendizagem prática e assistência adequada é prejudicada pela situação de emergência em si e consequente a segurança do paciente. Por este motivo, atividades de educação continuada baseadas na metodologia de simulação clínica permitem a capacitação dos profissionais médicos e enfermeiros segundo as principais diretrizes internacionais em situações de emergências e urgências, garantindo um ambiente seguro, com possibilidade de repetição e consequente retenção do conhecimento de forma motivadora ${ }^{7,8}$.

Diversos estudos apontam os fatores psicológicos da simulação em relação ao estresse, evidenciando as diversas possibilidade de sua utilização no sentido da retenção do conhecimento. A simulação tem se mostrado efetiva ferramenta de ensino para capacitação profissional no âmbito das emergências clínicas, apontando para um resultado promissor com foco na segurança do paciente ${ }^{9}$. Nesse sentido, o emprego da simulação clínica na Educação Continuada é relevante na aprendizagem de processos cognitivos, adequação de casos clínicos específicos da especialidade a ser treinada, desenvolvimento de trabalho em equipe e demais habilidades comportamentais, além da possível exposição a situações raras que exijam condutas imediatas ${ }^{8}$.

Com base na Política Nacional de Educação Permanente em Saúde (PNEPS), que tem como um dos pontos cruciais a regionalização do Sistema Único de Saúde (SUS), a implementação de diferentes estratégias educacionais promovem a potencialização de melhorias das ações dos cuidados em saúde e que devem partir das necessidades do território².

\section{Objetivo}

Descrever o processo de formação desenvolvido por meio da Educação Continuada em atendimentos de urgências e emergências, na qualificação dos profissionais médicos e enfermeiros na Coordenadoria Regional de Saúde Centro do Município de São Paulo.

\section{Metodologia}

Estudo descritivo do tipo relato de experiência, com abordagem qualitativa. As estratégias de ensino-aprendizagem foram realizadas no ambiente "hospital simulado" da Faculdade de Medicina da Universidade Cidade de São Paulo no período de 2018 a 2019.

Foram utilizadas metodologias ativas de aprendizagem, com base na simulação clínica e na aprendizagem baseada em equipe, envolvendo médicos e enfermeiros dos serviços que compõe a Rede de Urgência e Emergência da Região Central do Município de São Paulo.

Esta região possui diversas particularidades e iniquidades sociais, contando com importantes centros comerciais e financeiros, de modo que circulam neste território cerca de 3.000 .000 pessoas/dia. Como moradores da área, somam-se mais de 431 mil pessoas, representando $3,8 \%$ da população total do Município ${ }^{10,11}$. Esta região possui a maior cena de uso de substâncias psicoativas do município e mais da metade das pessoas em situação de rua ${ }^{3}$. Tanto o planejamento das ações em saúde como a organização dos equipamentos neste território é um desafio constante. As unidades da Rede de Atenção a Saúde destinadas ao atendimento de situações de urgência e emergência são quatro Ambulatórios de Assistência Médica e um Pronto Socorro ${ }^{10,11}$.

As atividades foram fundamentadas em três eixos temáticos: a) Atenção à Saúde: cuidado integral à saúde individual e às necessidades coletivas de saúde; b) Gestão em Saúde: gestão de recursos e de cuidado; e c) Educação em Saúde. Para análise dos dados, utilizou-se a metodologia de observação participante, que consiste em uma técnica de investigação social em que o observador partilha, na medida em que as circunstâncias o permitam, as atividades, as ocasiões, os interesses e os afetos de um grupo de pessoas $\frac{12}{}$. 


\section{Resultados e discussão}

Para levantamento dos dados relacionados a necessidade de educação continuada das equipes foram realizadas reuniões nos equipamentos de saúde da Região Central de São Paulo. O responsável local pelo equipamento de saúde realizou encontros com as equipes para um mapeamento da necessidade autorreferida de treinamento e epidemiologia das doenças e agravos mais prevalentes. Após estes encontros, foram realizados alguns debates com todos os responsáveis locais de equipamentos do Centro de São Paulo e um facilitador de metodologias ativas de aprendizagem, permeando a troca de experiências e avaliação entrepares. O resultado destes encontros foram as construções coletivas do Plano Anual de Educação Permanente (PLAMEP), a partir do olhar do trabalhador da saúde. Estes encontros aconteceram com um facilitador da equipe de educação permanente, tendo como eixos teóricos metodológicos: o Programa Nacional de Melhora do Acesso e Qualidade na Atenção Básica (PMAQ-AB-MS), as diretrizes assistenciais dos Conselhos de Classe relacionados e as diretrizes próprias do município de São Paulo.

A primeira fase da capacitação foi realizada em 2018 e contou com a participação de 20 médicos de diferentes especialidades de atendimento ligados à urgência e emergência. Atendimentos cardiovasculares, pediátricos, obstétricos, além do paciente politraumatizado foram priorizados, utilizando simuladores de alta fidelidade com recurso áudio visual, manequins e laboratório de técnica cirúrgica para procedimentos hands on. No Quadro 1 estão descritos em detalhes as temáticas e metodologias utilizadas.

Quadro 1. Atividade simulada para educação em saúde de médicos com foco no atendimento às situações de urgência e emergência clínica

\begin{tabular}{|c|c|c|}
\hline Temática & Metodologia & Recursos Utilizados \\
\hline $\begin{array}{l}\text { Manipulação de vias aéreas: } \\
\text { Dispositivos de vias aéreas não } \\
\text { invasivos e intubação orotraqueal }\end{array}$ & $\begin{array}{l}\text { Task Trainer (treinamento de } \\
\text { habilidades específicas) }\end{array}$ & $\begin{array}{l}\text { Manequins e softwares específicos } \\
\text { para uso desde cateter nasal, } \\
\text { máscara de venturi, máscara } \\
\text { laringea e intubação orotraqueal. }\end{array}$ \\
\hline Punção intraóssea & $\begin{array}{l}\text { Task Trainer (treinamento de } \\
\text { habilidades específicas) }\end{array}$ & $\begin{array}{l}\text { Uso do laboratório de técnica } \\
\text { cirúrgica com peça animal e } \\
\text { manequim específico. }\end{array}$ \\
\hline Suporte Básico de Vida & $\begin{array}{l}\text { Prática Deliberada em Ciclos } \\
\text { Rápidos, uso de feedback imediato. }\end{array}$ & $\begin{array}{l}\text { Reconhecimento e manejo inicial a } \\
\text { parada cardiorrespiratória e } \\
\text { utilização do desfibrilador externo } \\
\text { automático. }\end{array}$ \\
\hline $\begin{array}{l}\text { Politraumatizado, Eclâmpsia seguido } \\
\text { de Reanimação Neonatal, manejo do } \\
\text { infarto agudo do miocárdio com } \\
\text { elevação de ST seguido de parada } \\
\text { cardiorrespiratória e Choque Séptico } \\
\text { na criança. }\end{array}$ & $\begin{array}{l}\text { Cenários avançados com simulação } \\
\text { padrão seguidos de discussão no } \\
\text { estilo debriefing. }\end{array}$ & $\begin{array}{l}\text { Salas e simuladores específicos para } \\
\text { cada especialidade com recurso } \\
\text { áudio visual para otimizar a } \\
\text { discussão posterior. }\end{array}$ \\
\hline
\end{tabular}

A estratégia da simulação clínica pode variar de acordo com a demanda, neste contexto não apenas considerar o local de trabalho destes profissionais para adequar os casos clínicos a serem treinados, mas como garantir que haja uniformização do conteúdo do grupo a ser capacitado4. Neste contexto, as atividades foram divididas entre task trainer para o resgate de procedimentos hands on através de manequins, práticas deliberadas de ciclo rápido onde há feedback imediato ao longo do atendimento aos pacientes e por fim a simulação padrão onde o atendimento não sofre interrupções e será discutido em sala de debriefing com apoio do recurso áudio visual.

A segunda fase das capacitações foram realizadas também em 2018 e contou com a participação de 16 enfermeiros como foco no suporte básico à vida para profissionais de saúde, que contemplou o reconhecimento e o manejo a parada cardiorrespiratória adulto e pediátrico, técnicas adequadas para manipulação de vias aéreas, além das manobras de engasgo também em adultos e crianças. Neste curso foram utilizados também os simuladores avançados para cenários desenvolvidos dentro do contexto de rotina destes profissionais. 
Figura 1. Simulador de alta fidelidade para cenário de paciente politraumatizado

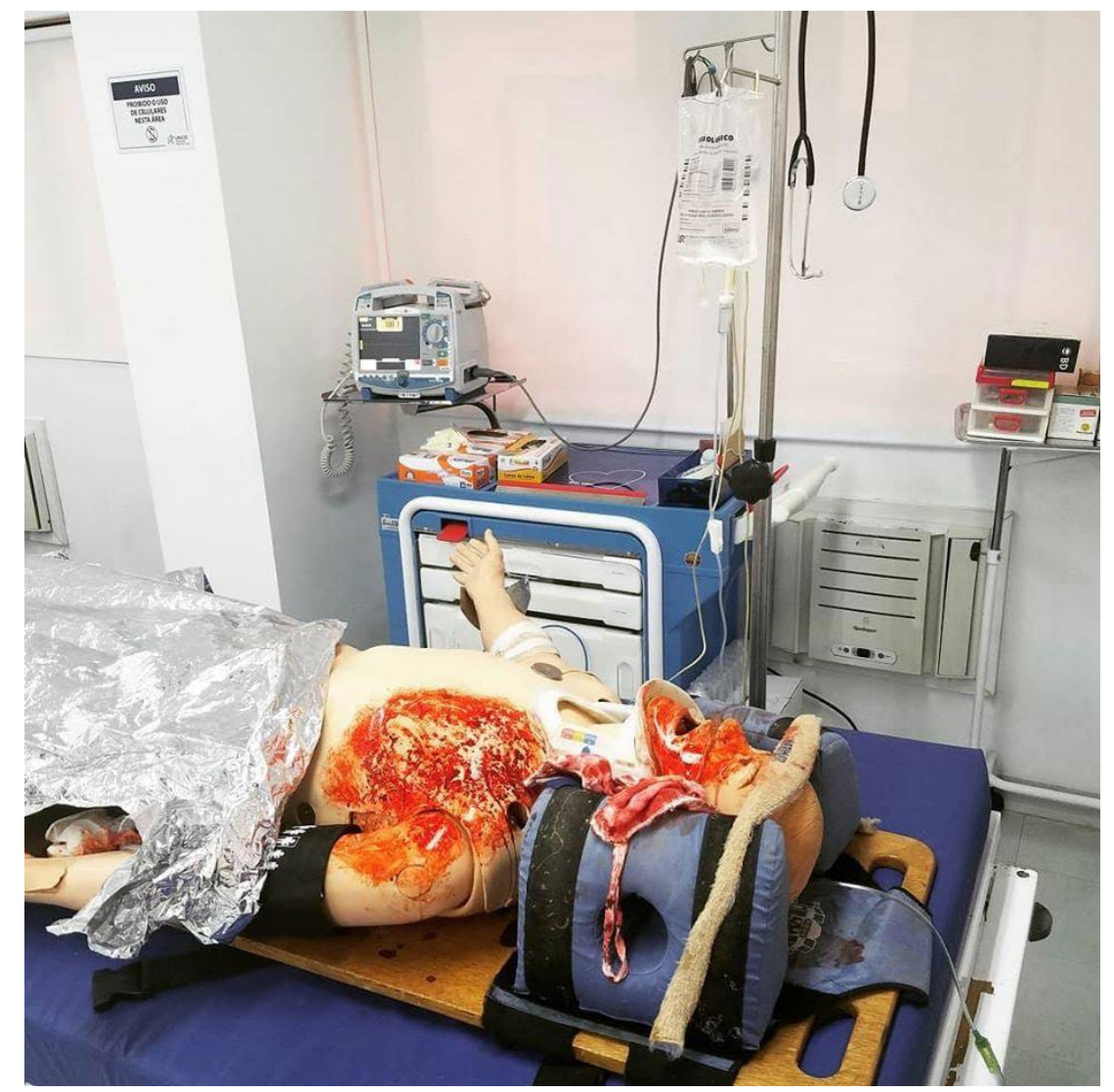

Considerando que os profissionais de saúde que atuam na atenção primária inevitavelmente podem atender pacientes em situações críticas, nas quais as condutas de reconhecimento e manejo imediato determinarão o prognóstico do paciente, em 2019, após a avaliação positiva dos resultados alcançados dos primeiros cursos e considerando as demandas existentes nas unidades, foi realizado um programa de formação para profissionais da atenção primária, com foco no manejo das situações de urgência e emergência clínicas.

O programa de formação foi desenvolvido em 5 módulos autônomos, contando com a participação de 66 profissionais, dentre enfermeiros e médicos. O objetivo foi rever, uniformizar e ampliar os conhecimentos fundamentais de situações de urgências e emergências na atenção primária, utilizando as diretrizes mais relevantes e atuais, ressaltando especialmente as abordagens imediatas e condutas até o momento da transferência do paciente considerando a dinâmica ambiente em questão. Baseado na discussão dos casos clínicos apoiado pela realização de técnicas hands on em manequins estáticos e simuladores, foram abordados os temas: Atendimento ao paciente politraumatizado conforme representado na figura 1, atualização em sepse no adulto e pediatria, neurologia clínica, Insuficiência Respiratória, Emergências Obstétricas e o manejo da descompensação no diabetes e suas condutas fundamentais na desidratação.

No intuito de complementar as atividades modulares, como estratégia para aprofundar o conhecimento teórico, também foram realizadas sessões de Aprendizagem Baseada em Equipe (ABE). Inicialmente, a cada módulo, foi aplicado um pré-teste individual com questões que abordavam aspectos fundamentais do tema. Após a realização das etapas de contextualização teórica e capacitação prática das técnicas, realizou-se discussões em grupos de casos voltados à realidade da atenção primária, nas quais os participantes deveriam decidir coletivamente as medidas que seriam adotadas, sendo finalizado com a correção conjunta dos mesmos e das questões do pré-teste, esclarecendo-se as dúvidas remanescentes. 
Um fator limitante para expansão e reprodutibilidade destes treinamentos são o espaço físico e todos os materiais e recursos necessários para realizar uma simulação clínica de alta fidelidade, desde robôs e manequins até recursos áudio visuais. Outro grande desafio são os facilitadores ou instrutores destas metodologias, uma vez que o sucesso desta atividade se deve, em grande parte, a um profissional habilitado educacionalmente. A telessimulação, ou seja, o caso clínico simulado de forma remota, tem expandido consideravelmente, mostrando-se uma alternativa viável para formação profissional13,14.

\section{Considerações finais}

As metodologias ativas de aprendizagem promovem o desenvolvimento do trabalho em equipe e interprofissional, sedimenta processos cognitivos, melhora as habilidades psicomotoras através de um ambiente participativo, com repetição e segurança, e quando bem definidos os objetivos e construídos de forma específica à realidade dos profissionais, promove o estímulo de melhoria e desenvolvimento de competências. Por meio da investigação direta da realidade a construção do conhecimento leva a uma efetiva compreensão dessa mesma realidade. A formulação e utilização dos mecanismos de gestão e avaliação baseados no planejamento estratégico e análise de indicadores de monitoramento e desempenho, propiciaram a criação de treinamentos baseados em metodologias ativas de aprendizagem com temáticas específicas direcionadas a estes grupos de profissionais. É importante ressaltar que os trabalhadores envolvidos, direta ou indiretamente, demonstraram engajamento durante o treinamento e se reconheceram como equipe, modificando seus processos de trabalho. Por outro lado, esta organização de educação continuada demanda tempo, logística e o emprego de diferentes tecnologias para tornar possível uma metodologia híbrida que considere os temas estratégicos do ponto de vista institucional e os temas construídos coletivamente a partir da ótica do trabaIho e necessidade do território. Dessa forma, sugere-se a realização de novos estudos para aprofundar a análise e a avaliação dos resultados práticos ligados diretamente ao paciente.

\section{Contribuições dos autores}

Todos os autores participaram de todas as etapas da pesquisa, redação, revisão e aprovação final do relato.

\section{Conflitos de interesses}

Nenhum conflito financeiro, legal ou político envolvendo terceiros (governo, empresas e fundações privadas, etc.) foi declarado para nenhum aspecto do trabalho submetido (incluindo, mas não se limitando a subvenções e financiamentos, participação em conselho consultivo, desenho de estudo, preparação de manuscrito, análise estatística, etc.).

\section{Referências}

1. Neves GBC, Andreto LM, Oliveira CR, Figueira MCS. Opinião dos enfermeiros sobre educação permanente em um hospital público. Rev Enferm UFPE. 2016;10(5):1625-1634. doi: 10.5205/ reuol.9003-78704-1-SM.1005201608

2. Brasil. Ministério da Saúde. Secretaria de Gestão do Trabalho e da Educação na Saúde. Departamento de Gestão da Educação na Saúde. Política Nacional de Educação Permanente em Saúde: o que se tem produzido para o seu fortalecimento? / Ministério da Saúde, Secretaria de Gestão do Trabalho e da Educação na Saúde, Departamento de Gestão da Educação na Saúde - 1. ed. rev. Brasília: Ministério da Saúde; 2018.

3. Kern DE, Thomas PA, Hughes MT. Curriculum Development for Medical Education: A six-step Approach. 2nd ed. Baltimore: Johns Hopkins University Press; 2009.

4. Michaelsen LK, Knight AB, Fink LD. Team based learning. Sterling: Stylus Publishing; 2004.

5. Johnson C. Team-Based Learning for Health Professions Education: A Guide to Using Small Groups for Improving Learning. J Chiropr Educ. 2009;23(1):47-8.

6. Bollela VR, Senger MH, Tourinho FSV, Amaral E. Team-based learning: from theory to practice. Medicina. 2014;47(3):293-300. doi: 10.11606/issn.2176-7262.v47i3p293-300

7. Brandão CFS, Carvalho-Filho MA, Cecilio-Fernandes D. Simulation centers and pedagogical planning: Two sides of the same coin. Sci Med. 2018;28(1):ID28709. doi: 10.15448/19806108.2018.1.28709

8. Cecilio-Fernandes D, Brandão CFS, Oliveira DLC, Fernandes GCVR, Tio RA. Additional simulation training: does it affect students' knowledge acquisition and retention? BMJ Simul Technol Enhanc Learn. 2019;5(3):140-43. doi: 10.1136/ bmjstel-2018-000312 
9. Dias RD. Nível de estresse durante o atendimento às emergências: comparação entre realidade e cenários simulados [tese]. São Paulo: Faculdade de Medicina; 2015.

10. Prefeitura da Cidade de São Paulo. Mapa Digital da Cidade de São Paulo [Internet]. [acesso em 2020 set 18]. Disponível em: http://geosampa.prefeitura.sp.gov.br/PaginasPublicas/_SBC.aspx

11. Prefeitura da Cidade de São Paulo. Centro de Pesquisa e Memória Técnica [Internet]. 2020. [acesso em 2020 set 18]. Disponível em: https://www.prefeitura.sp.gov.br/cidade/ secretarias/assistencia_social/observatorio_social/pesquisas/ index.php?p=18626
12. Anguera MT. La observación. En: C. Moreno Rosset, editor. Evaluación psicológica. Concepto, proceso y aplicación en las áreas del desarrollo y de la inteligência. Madrid: Sanz y Torres; 2003.

13. McCoy CE, Sayegh J, Alrabah R, Yarris LM. Telesimulation: An Innovative Tool for Health Professions Education. AEM Educ Train. 2017;1(2):132-136. doi: 10.1002/aet2.10015

14. Papanagnou D. Telesimulation: A Paradigm Shift for Simulation Education. AEM Educ Train. 2017;1(2):137-139. doi: 10.1002/aet2.10032 